\title{
Gaining and Maintaining Employee Trust in Academia: The Cultural Setting Perspective
}

\author{
Yvette Ferguson Arthur \\ School of Management, University of Derby, United Kingdom \\ John Kingsley Arthur \\ Computer Science Department, Valley View University, Ghana \\ Kofi Sarpong Adu-Manu \\ Computer Science Department, Valley View University, Ghana
}

\begin{abstract}
Good working relations between employers and employees are essential for the growth of an organization. One of the very key carriers in building this good relation is employee trust. Employee and its dynamics have been widely considered for institutions such as finance, auditing, sports, but not academia. Also, the majority of the current work has focused on only one region and its social work setting on employee trust. To this extent, this research work aims to propose a theoretical framework bringing to light the factors for gaining and maintaining employee trust in academia. A comparative study is done using the case of the University of Derby (UK) and Valley View University (Ghana). Both quantitative and qualitative methods are used to compare the responses from the two countries. The findings revealed that general trust is influenced by a lot of drivers like the consistency of managers, length of work, and place of work. There appeared to be some statistical evidence of the difference between the two countries. Nevertheless, it did not reveal the differences in culture at work.
\end{abstract}

Keywords: Employee Trust, Foreign fund, Organizational performance, Leadership, Culture.

DOI: $10.7176 /$ RJFA/10-22-06

Publication date: November $30^{\text {th }} 2019$

\section{Introduction}

Employee trust is one of the essential drivers of the success of an organization. When employees trust their employers, their output is much higher than the reverse. The Government of Ghana had hastened the timing for the achievement of the Millennium Development Goal (MDG) on Achieving Universal Primary Education(Amoateng, 2009), given a clear indicator that the harmonious growth of the educational sector is of keen interest in Ghana. According to Clark(2011), higher education is central to economic growth, and the United Kingdom (UK) has one of the most successful higher education systems in the world. However, over the last decade, studies have shown that workplace trust is diminishing, and the educational sector is no exemption (Lema, 2011).

Some of the researches done in this problem area are that of Lema(2011), Pahl(2008), STUC(2009), Dan(1992), Boe(2002), and Askvik(2010). However, all of the earlier works did not consider the effects of the trust factors across different working cultures. Therefore, this research examines whether factors affecting employee trust are applicable across large organizations with different cultural settings, specifically in Ghana and the United Kingdom.

\section{Literature Review}

\subsection{Trust and Issues of Cultural Difference}

Cultural differences have a significant role in the creation and maintaining of trust. Trust means differently to different people around the world. In China, the Arab, and the Latin American countries, professional interactions are determined by the relationships built over time. In these countries, it takes much time to develop trust, unlike with other cultures (Asherman et al.,2000). In a country like the United States of America, strangers assume they both have good intentions, and hence, the assumption of trust is generally high unless otherwise proven wrong.

\subsection{Cultural Influences on Leadership and the Organization}

Leadership expectation, behavior, as well as status, are influenced by the cultural forces of the countries where the leaders work. In countries like the USA, England, France, and Russia are keen on the term leadership concerning politics and the organizational arena (House et al., 2000). The Americans have two kinds of leaders; autonomous leadership and a leader who have the right to delegate their authority to subordinates or employees. They appreciate leaders that are bold, confident, risk-takers. The Dutch, on the other hand, stresses more on egalitarianism and are particular about their leadership values. It goes to the extent of children not admitting their 
parent's leadership positions to their course mates.

\subsection{Theory of Cultural Difference in Management}

Khastar et al. (2011) provided a framework for the effects of countries' cultural beliefs on organizations' culture. Their work relied on Hofstede's theory of organizational culture, which is categorized into five(5) dimensions; power distance, uncertainty avoidance, individualism versus collectivism, Masculinity versus femininity. All of which are regional dependent factors influencing employee trust.

\subsection{Proposed Theoretical Framework for Employee Trust}

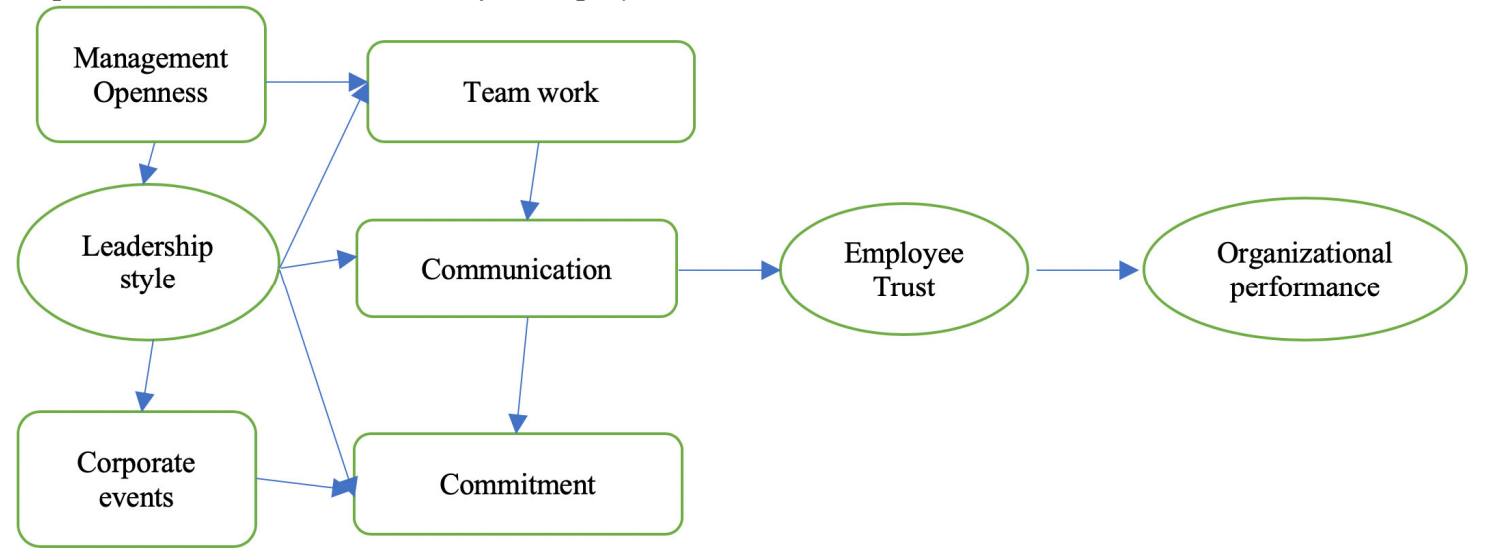

Fig 1: Theoretical Model

The model above shows that leadership style is inter-linked with management openness and honesty as well as the organization of corporate events by management. However, leadership styles will influence teamwork, communication in the organization, and commitment of employees. All these factors, on the right hand, will come together to affect the level of employee trust, which will determine the performance of an organization (Asherman et al., 2000). It also shows how the national culture of different countries influences all the elements in the model. Therefore, the subsequent sections discuss the questions of the factors on the model for its examined understanding.

\section{Methodology}

In this research work, both quantitative and qualitative approaches are used. The cross-sectional and case A dual strategy is adopted for the research, namely, the cross-sectional and case study. The cross-sectional study will be used to gather data from employees in different cultures and countries but in the same period. Two different countries (UK and Ghana) were used to ascertain how their opinions on the topic differ.

\subsection{Population and Sample}

The group of interest to the researcher was employees of the Business School at the University of Derby and the School of Business, Valley View University. The target population was supervisors of independent postgraduate students as well as undergraduate module leaders. The questionnaires were administered to all members of the sample. This selection is made on purposive or judgmental sampling for the comparative nature of the study.

\subsection{Data Collection and Data Analysis}

Both primary and secondary data are collected; that is from literature (the articles, and books, etc.) and admiration of online questionnaire form to respondents to fill. Questions like how the employee will define trust, whether trust is important, what factors affect trust in the organizations, will be asked. The questions will produce quantitative data that will be highly specific and precise. However, the qualitative data derived was converted into quantitative data for analysis. The researcher will use the Statistical Product for Service Solutions (SPSS) for the data analysis.

\subsection{Questionnaire Design}

The questionnaire is divided into two(2) sections. The section captures the background information such as age, gender about the respondents. The section will also contain questions like years of experience, level of qualification and place of work. The section 2 asks questions about the effects diversity of cultures on employee trust. This section had the respondents answer questions on what strategies would affect their trust for their managers. Respondents will be given a set of options of which they will have to rate on a scale of 1-5, that is, 1 representing essential and 5 representing not essential. 


\section{Analysis and Discussion of Findings}

To analyze the evidence of cultural differences between the University of Derby, UK, and Valley View University. Figure 2, a question of "My trust in my manager is affected by my manager organizing university staff and workers social outings"? is asked.

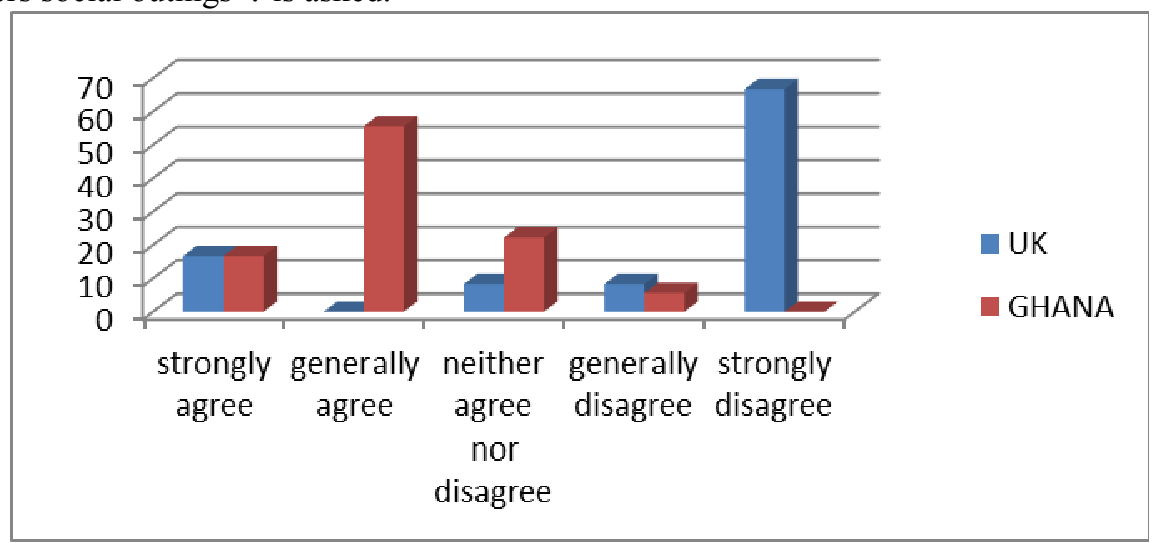

Figure 2: My trust in my manager is affected by my manager organizing university staff and workers social outings

Ho- The responses are the same

H1 - The responses are different

Figure 2 represents Valley View University School of Business(VVUSB) and Derby Business School(DoB) employee perception of whether trust in their manager is affected by their managers organizing staff social outings. The responses show that the majority of the VVUSB (44.4\%) disagree, as against $75 \%$ of DoB that agree. This shows that there is a statistical difference between the two countries in respect to their perception of this driver of behavior. Hence the Ghana and UK perception of the statement above is different. Thus H1 is kept and reject Ho.

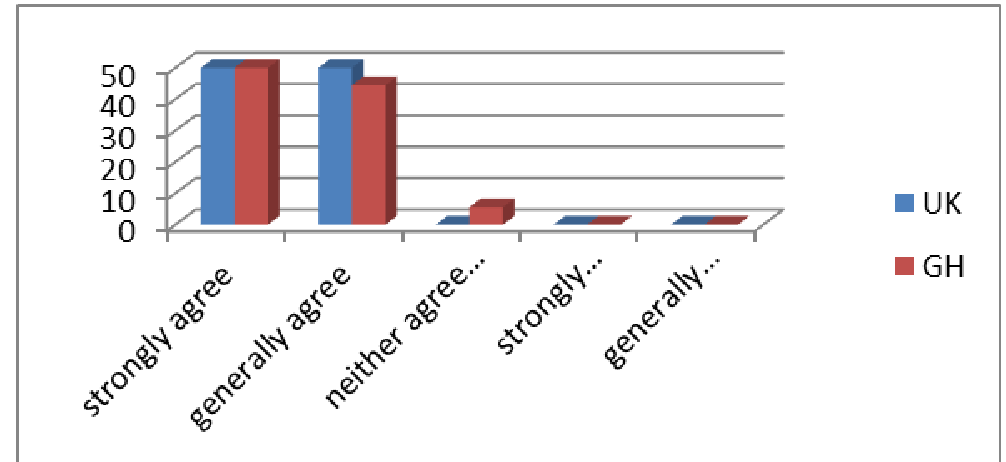

Figure 3: My trust in my manager is affected by being treated equally by my manager

Ho- The responses are the same

H1- The responses are different

From figure 3 above, both DoB and VVUSB employees agree that 'my trust in my manager is affected by being treated equally by my manager with no visible form of partiality'. The responses show that the majority agree with the statement. The VVUSB employees, although most agree to the statement, have a few employees who seem to be indecisive and others who disagree. From the figure, it shows that there is no statistical difference between the two countries, and hence we accept the $\mathrm{H} 0$ and reject the $\mathrm{H} 1$. 


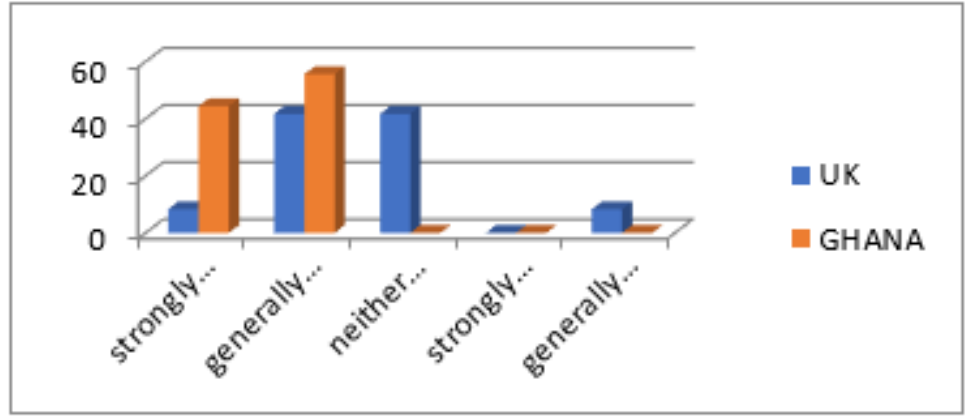

Figure 4: My trust in my manager is affected by team appraisal rather than individual

H0- The responses are the same

H1- The responses are different

Figure $4(33.4 \%)$ shows that the majority of employees in the DoB agree on the perception that their trust in their manager is affected by the team been praised by their manager rather than individuals. While an equal proportion generally agrees, some also have doubts about this statement (neither agree nor disagree). The VVUSB School of business employees, on the other hand (Ghana), all agreed to this statement. Although some of the employees from the Derby business school have doubts, the majority of them agreed. Hence we keep H0 and reject $\mathrm{H} 1$.

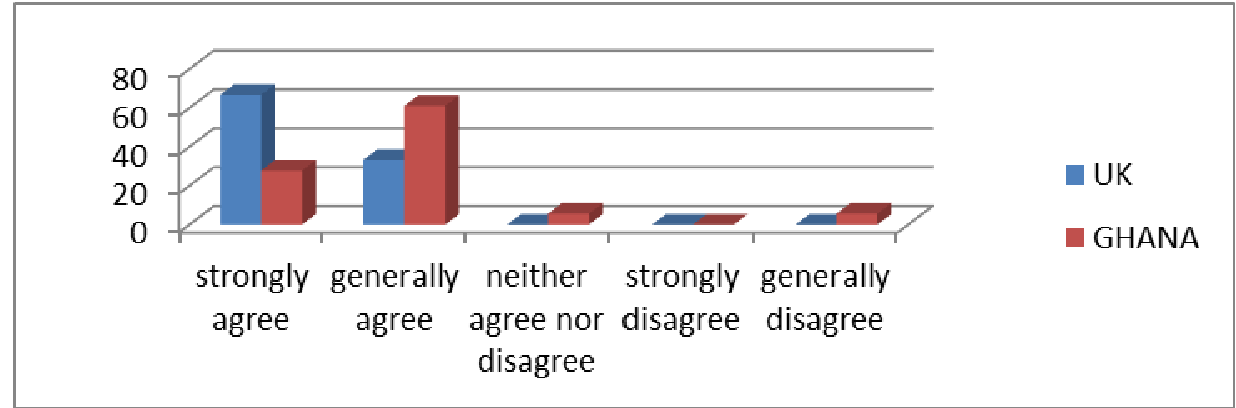

Figure 5: My trust in my manager is affected by being assigned similar work load

H0- The responses are the same

H1 - The responses are different

From the figure 5 above, both VVUSB and DoB agree that their trust in their manager is affected by being assigned a similar workload by their manager to others in a similar position in their university. But a small proportion of the employees in the VVUSB are indecisive, while others generally disagree. The H0 hypothesis is rejected, and the $\mathrm{H} 1$ is accepted. Hence there is a difference in the responses from the two countries.

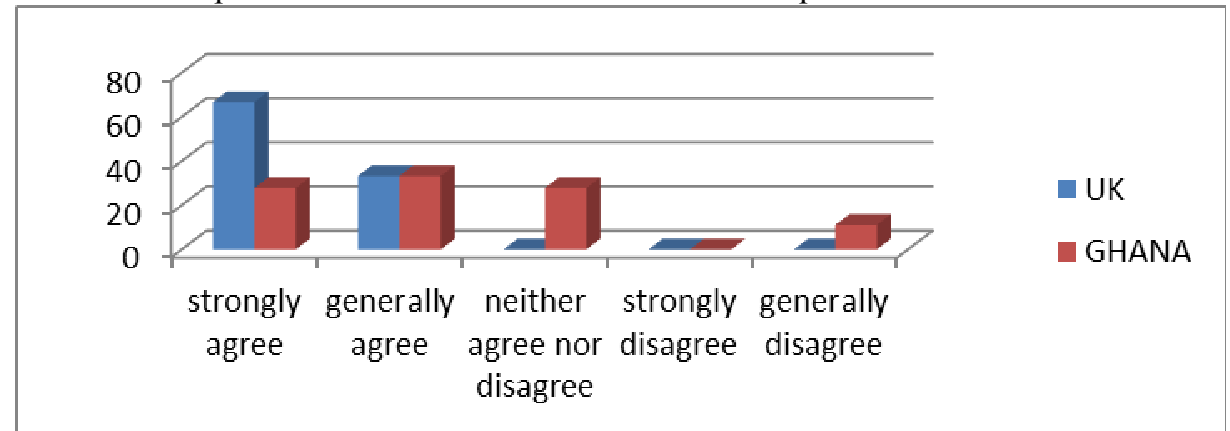

Figure 6: My trust in my manager is affected by my manager's consistent approach

H0- The responses are the same

H1 - The responses are different

Figure 6 shows the perception of employees in VVUSB and DoB on the statement that 'My trust in my manager is affected by my manager having a consistent approach.' The larger sample of employees from DoB $(55.6 \%)$ agrees to this statement while a smaller sample $(11.1 \%)$ neither agrees nor disagrees. The employees from VVUBS, on the other hand, have a different view. That is, $61.1 \%$ agreed to this statement while $27.8 \%$ neither agree nor disagree, and $11.1 \%$ disagree. Hence, the H0 hypothesis is rejected, but the H1 is accepted. This implies that there is a statistical difference between the two countries' responses. 


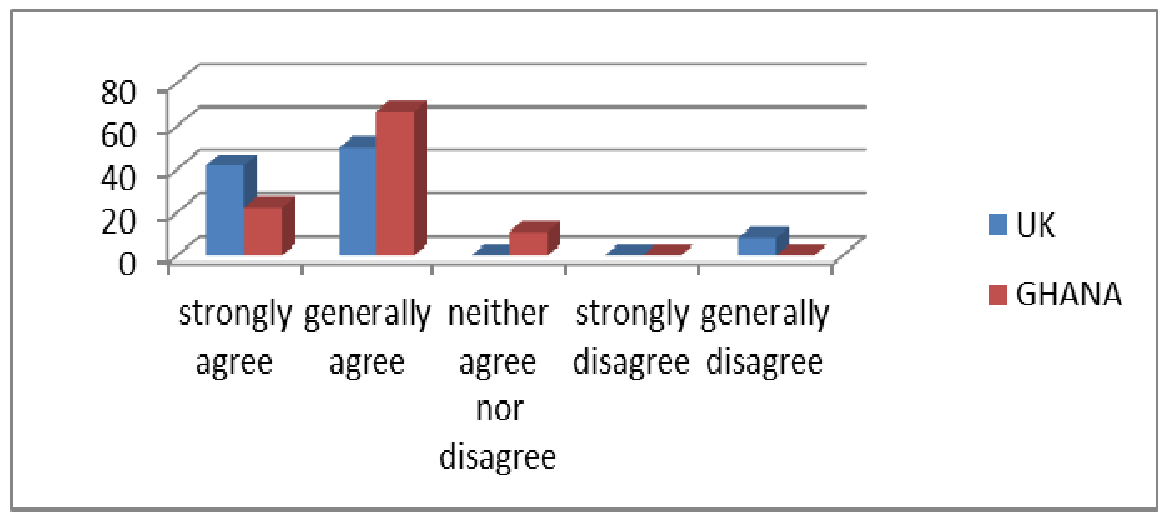

Figure 7: My trust in my manager is affected by individual employee circumstance consideration

H0- The responses are the same

H1- The responses are different

Figure 7 above shows the perception of employees' trust in their managers and how it is affected by their managers considering their employee circumstances. Figure 7 reveals that employees in the DoB strongly agree with this statement, with a small percentage (5.6\%) disagreeing. The VVUSB, on the other hand, has an average number strongly agree (22.2\%), and $66.7 \%$ generally agreeing. A percentage of $11.1 \%$ neither agrees nor disagrees. This data shows that the employees from the United Kingdom's perception of trust are highly influenced by managers considering individuals employee circumstances. Whereas the employees from Ghana generally agree to this but not strongly agree. Hence, $\mathrm{H} 0$ will be accepted, and the $\mathrm{H} 1$ rejected.

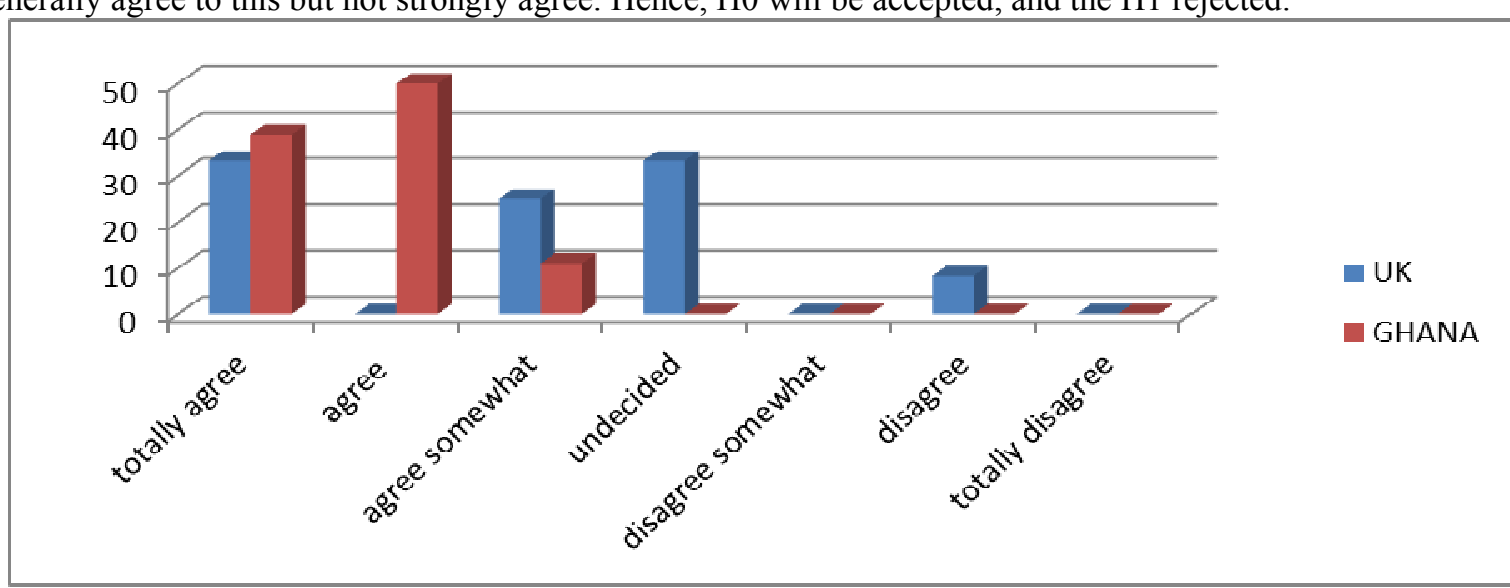

Figure 8: Low employee trust leads to increasing rate of employee turnover

H0- The responses are the same

H1- The responses are different

Figure 8 above shows the employee perception of low employee trust and how it leads to employee turnover. Most of the employees in DoB were indecisive about it. However, a higher proportion agreed with this, while a lesser portion disagreed. While in Ghana (VVU School of business), a higher percentage (88.9\%) agreed to this. The $\mathrm{H} 0$ is rejected, and $\mathrm{H} 1$ accepted. This means that there is a difference in the responses and hence there is also a statistical difference between the two countries. 


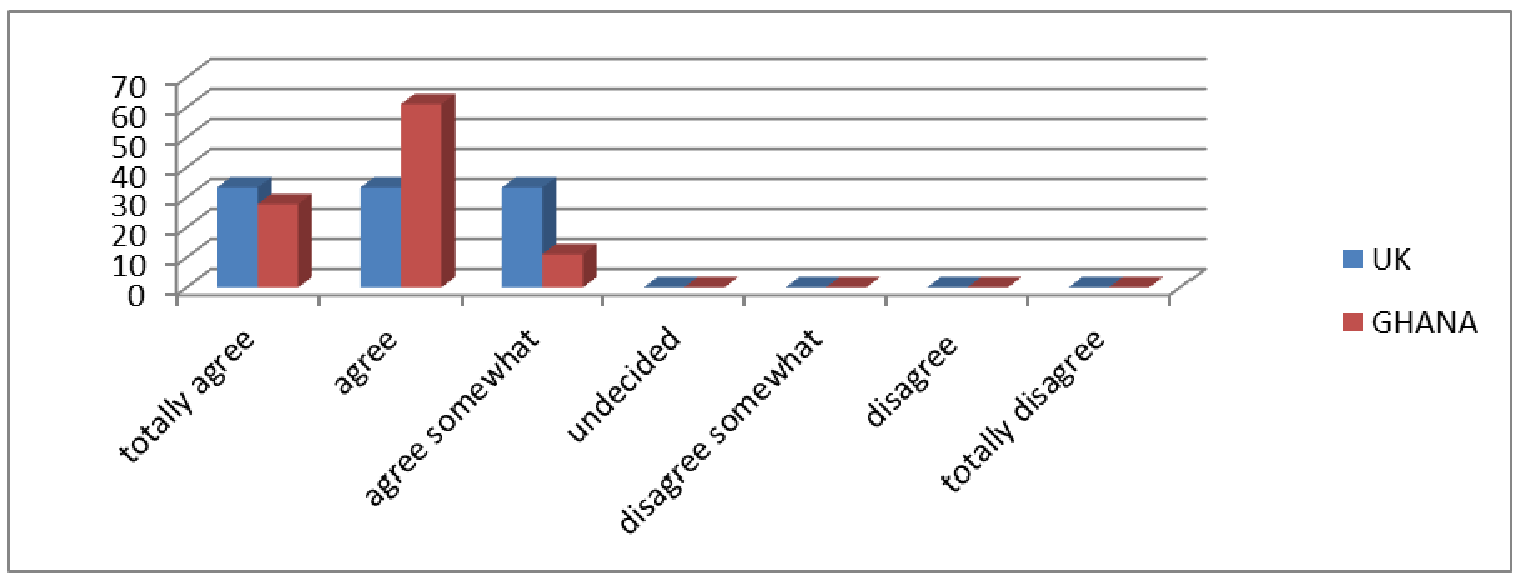

Figure 9: Leadership style influences my level of trust for my manager

H0- The responses are the same

H1- The responses are different

Figure 9, as shown above, represents the manager's leadership style and how it influences an employee's level of trust for their manager. The chart reveals that both respondents from the UK, DoB, and Ghana -VVUSB agree. None of the respondents, however, had some doubts about the statement or disagreed. This implies that there is a difference in both the responses from the two countries.

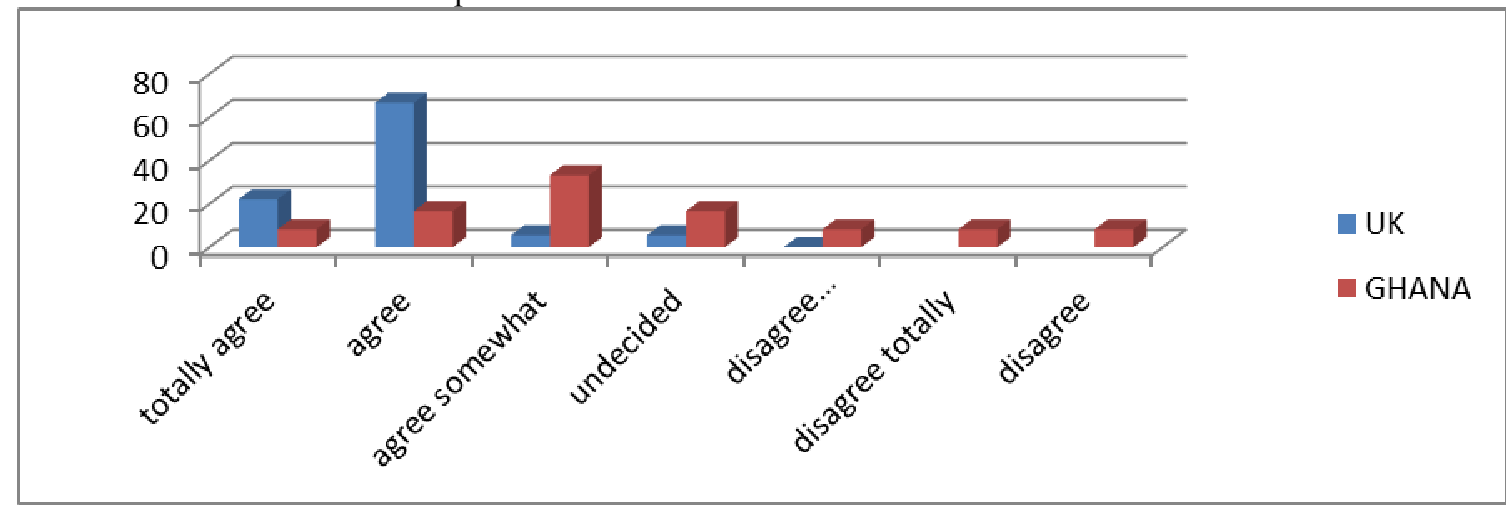

Figure 10: My work output is influenced by my trust in my manager

$\mathrm{H} 0$ - The responses are the same

H1- The responses are different

From figure 10 above, it can be seen that most of the respondents $(22.2 \%)$ from DoB agree somewhat to the perception that their trust influences their work output in their manager. VVUSB respondents, on the other hand, strongly agree to this with a higher percentage of $66.7 \%$. The null hypothesis H0 is rejected, and the H1 is accepted. This shows that there is a significant difference between the two countries and the responses gathered.

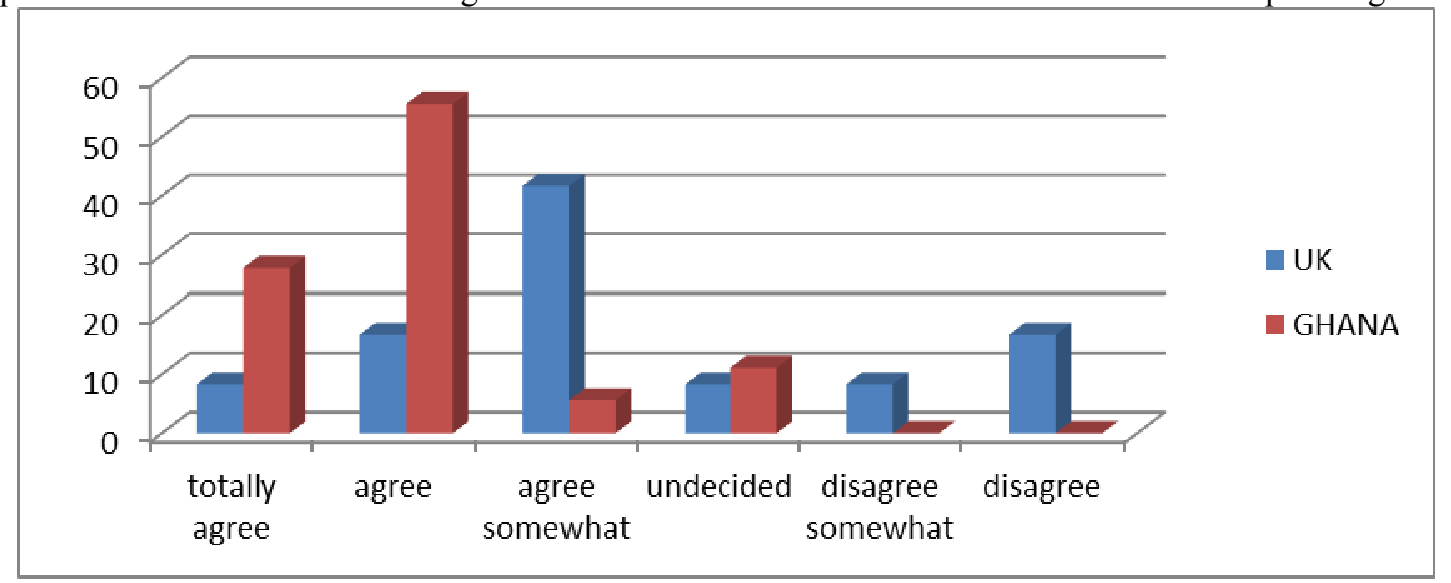

Figure11: My trust is influenced by my manager's recognition of my work input

H0- The responses are the same

H1- The responses are different

From figure 11, both $\operatorname{DoB}(\mathrm{UK})$ and $\operatorname{VVUSB}($ Ghana) have a percentage of employees agree that 'my trust in 
my manager is affected by being treated equally by my manager with no visible form of partiality' and other don't agree. The H1 will be accepted, showing evidence of statistical differences between the two universities.

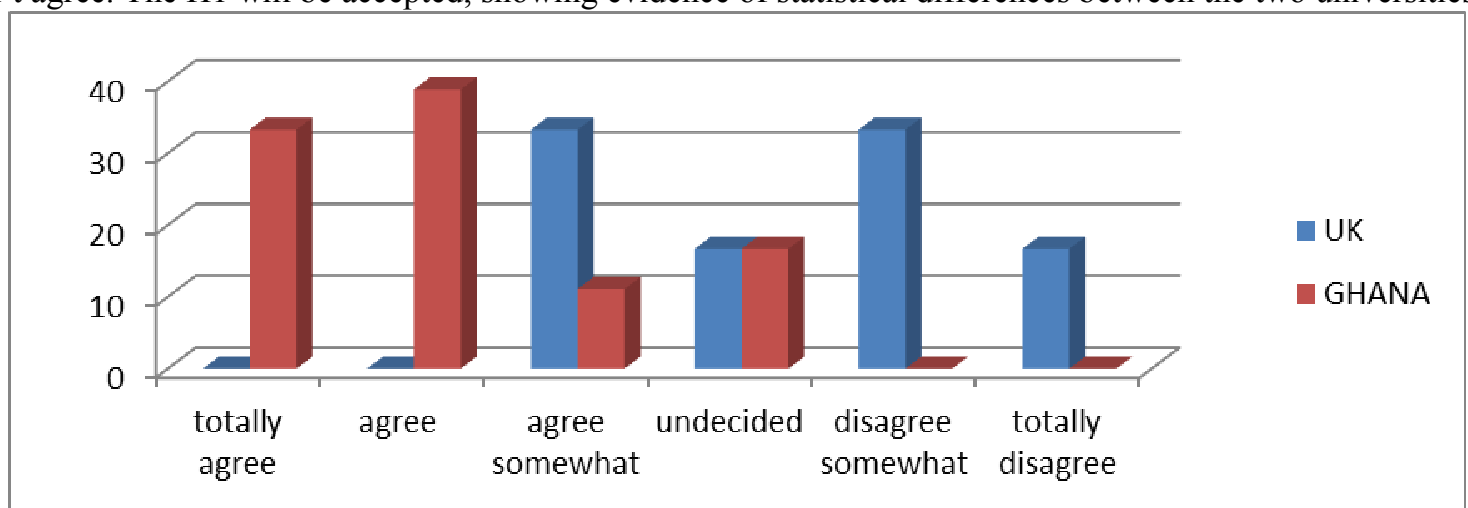

H0- The responses are the same

Figure 12: My work is linked to rewards from my manger

H1 - The responses are different

Figure 12 above shows that an employee's work is linked to their manager, giving them rewards. The respondents from VVUSB agree to this with a small sample of $16.7 \%$ undecided. The respondents from DoB strongly disagree with this with a total of $33.1 \%$. The $\mathrm{H} 1$ will be accepted, and this shows that there is a statistical difference between the two countries.

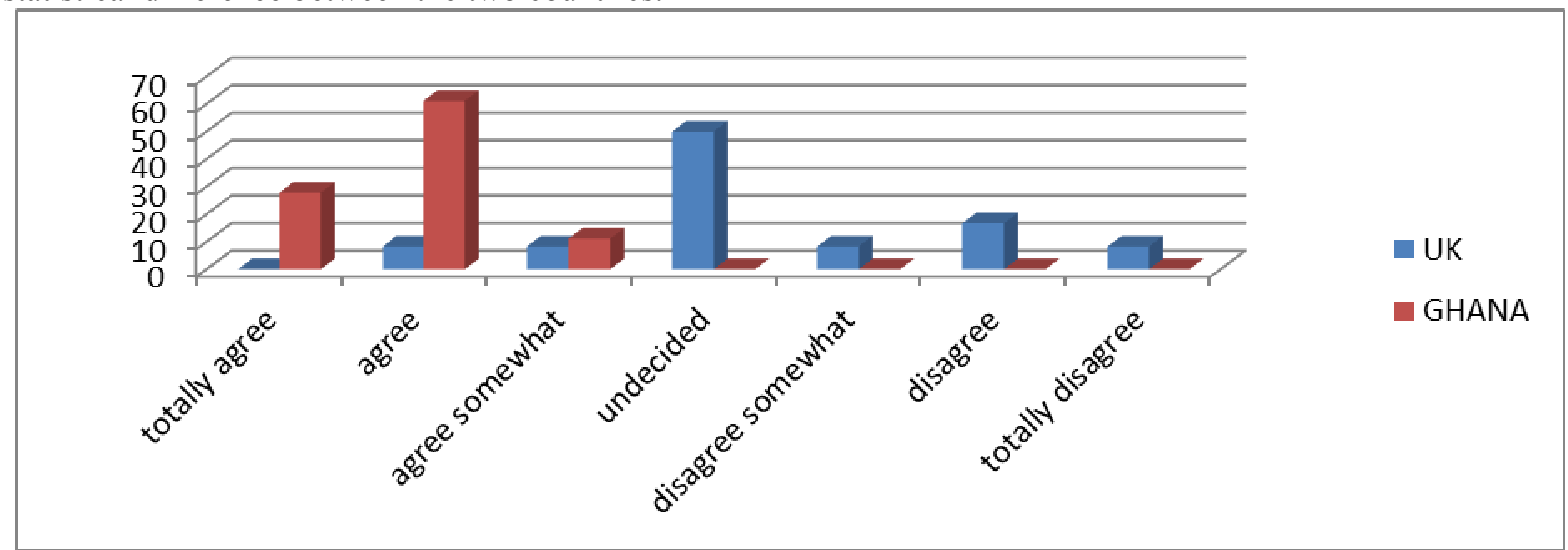

Figure 13: Staff teamwork influences my trust in my manager

$\mathrm{H} 0$ - The responses are the same

H1- The responses are different

Figure 13 shows employees' perception of how staff teamwork has a positive influence on an employee's level of trust in their managers. Most of the respondents from Derby business school $(22.2 \%)$ were undecided on this statement while the respondents from VVUSB agree. The null hypothesis (H0) will be rejected, and the H1 is accepted. The responses are the same, and there is statistical evidence of the difference between the two countries.

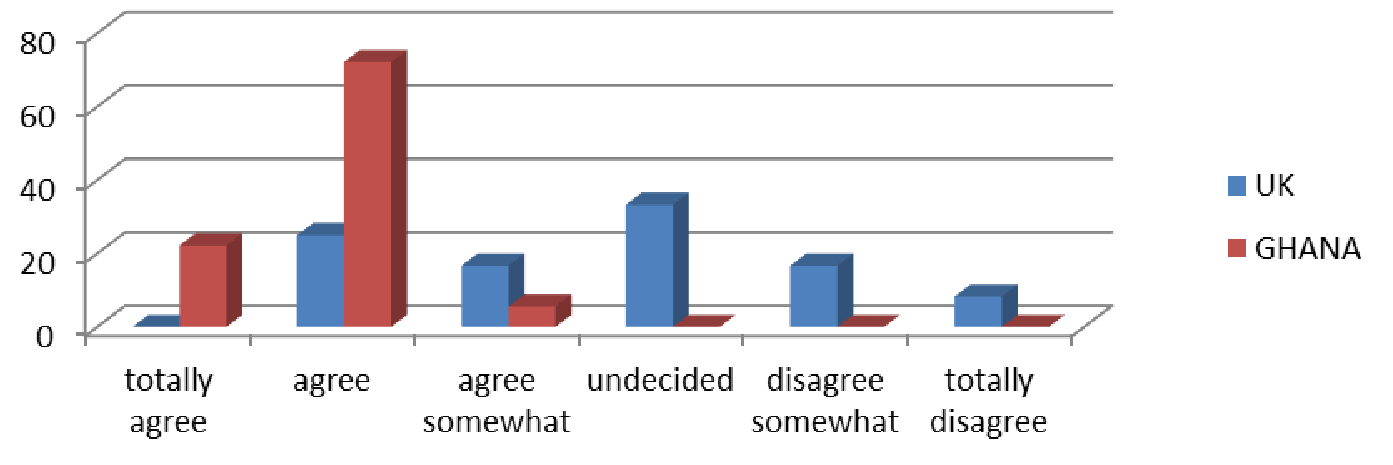

Figure 14: Communication is directly proportional to my trust in my manager

H0- The responses are the same

H1- The responses are different 
Figure 14 shows employees' perception of communication between staff members and their managers in their university and how it is directly proportional to their level of trust for their manager. Some of the respondents $(22.2 \%)$ from DoB were undecided whiles the respondents from Ghana(72.2\%) agree. The $\mathrm{H} 0$ is rejected, and $\mathrm{H} 1$ accepted.

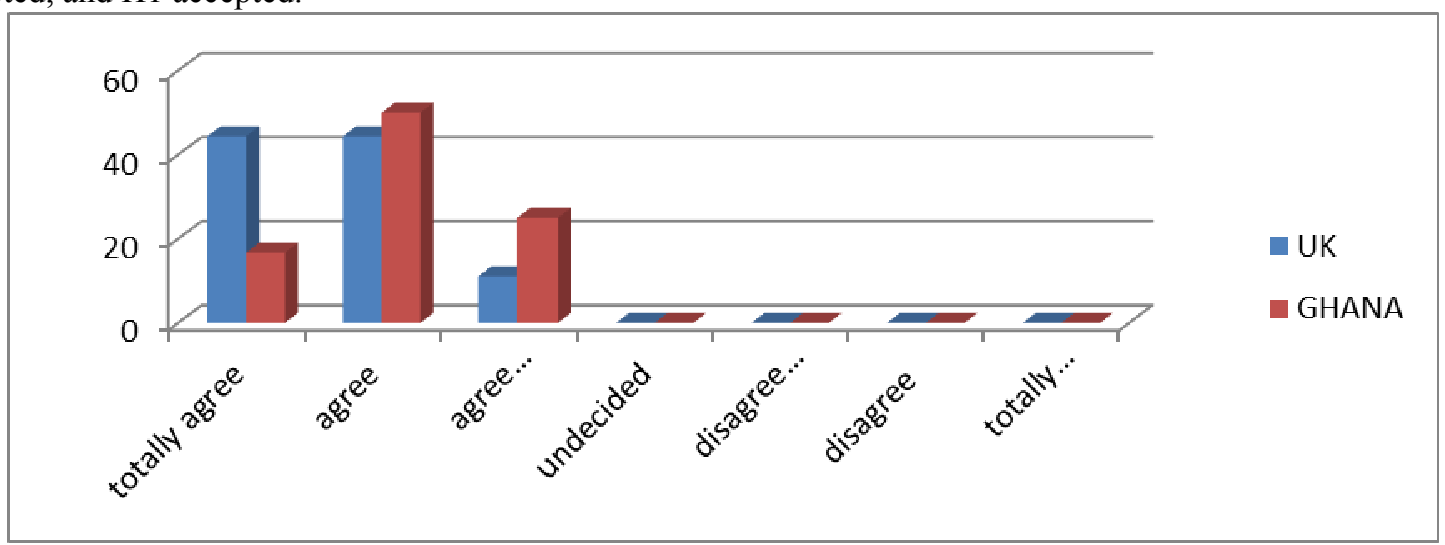

Figure15: My manager's behaviour and reputation affects my trust in him/her

H0- The responses are the same

H1 - The responses are different

From figure 15, both employees from Derby business school and VVU School of business agree that the manager's behavior and reputation affect an employee's trust. The H0 will be accepted, and the H1 will be rejected.

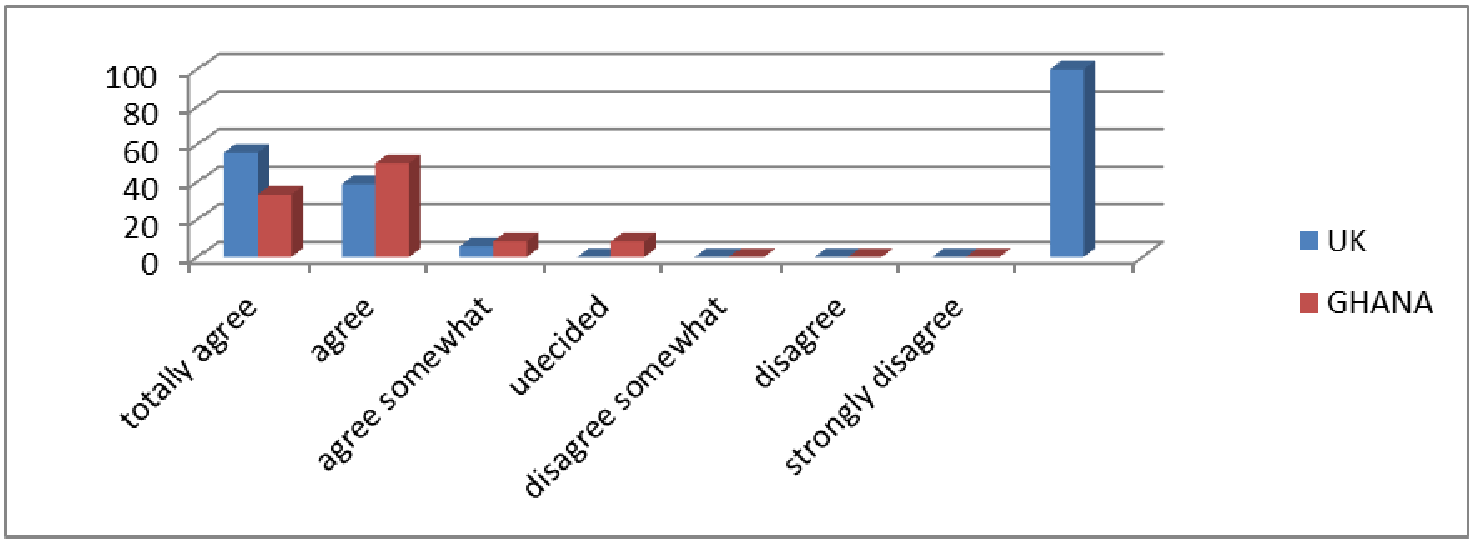

Figure 16: My manager's honesty and openness affects my trust

$\mathrm{H} 0$ - The responses are the same

H1- The responses are different

Figure 16 above shows that both employees from Derby business school and VVUSB agree that their manager's honesty and openness always influence their trust for their manager. This indicates that the employees from both countries (Ghana and the United Kingdom) have the same perception of how a manager's honesty and openness influence employee trust. There is some evidence of cultural differences from the respondents, but this is not clear. This is because, from the analysis of the first objective, it was seen that respondents from both Ghana and the United Kingdom turn to have a similar perception of the term 'trust.' Most of the responses either contradict some literature reviews or sides with it. But there is no visible form of cultural differences in the employees' perception of trust. 


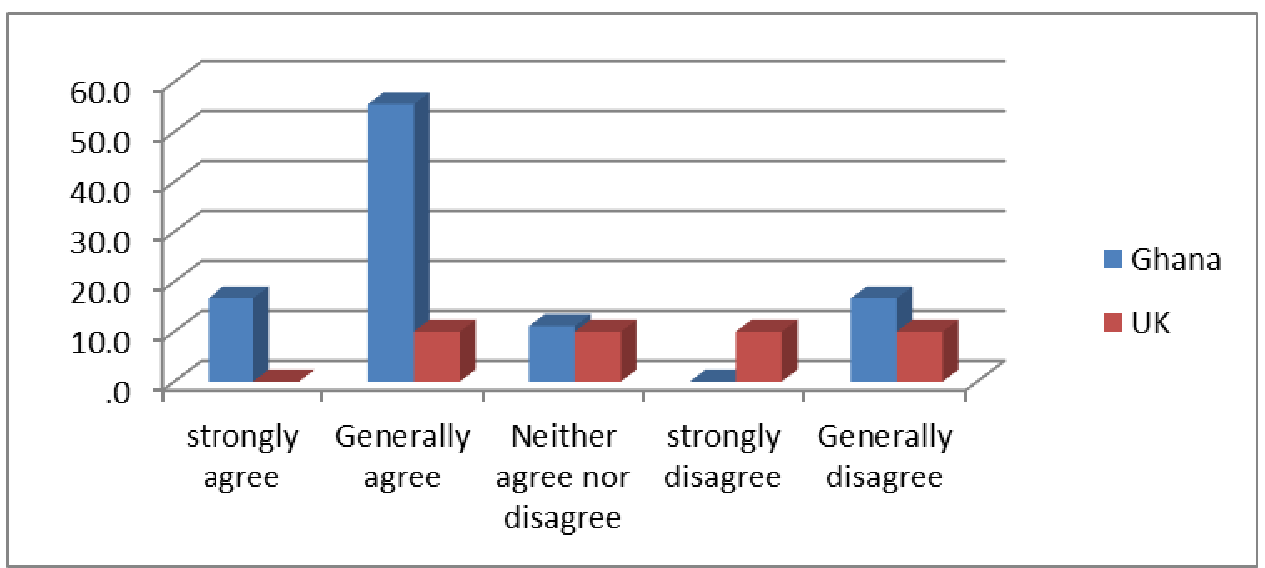

Figure 17: Culture analysis

orgottenuency table for the dependant variable is shown below. Thirteen (13) individuals generally agree to this. But it is might be cultural biased. Individuals are affected by the culture.

\section{Conclusion and Recommendation for further work}

For organizations to thrive in the new economy, trust has become an essential ingredient. The study revealed that other materials need to be part of the employee trust-building process. Management must create a trusted environment that is stimulating enough to restore trust.

The study expected to identify evidence of cultural differences between the perceptions of employees on trust in Ghana and the United Kingdom. From the comparison, it was determined that there is no visible difference when it comes to an employee's perception of employee trust in a larger organization (higher education). The research provided both theoretical explanations and empirical data on the impact of leadership on employee trust, strategies in gaining and maintaining trust.

There has been no study on gaining and maintaining employee trust in the educational sector with evidence of cultural differences. Hence, researchers can use this as pilot work and include more educational areas across countries to achieve some possible exciting outcomes.

\section{References}

Lema S. (2011). 5 Essentials to Build Employee Trust: Behaviors That Are Sometimes Forgotten Along the Way. Available:http://www.theglasshammer.com/news/2011/06/15/5-essentials-to-build-employee-trustbehaviors-that-are-sometimes-forgotten-along-the-way/.

Amoateng K. (2009). Right To Food In The Constitution of The Republic Of Ghana. Available: http://abibimman.blogspot.co.uk/.

Clark P. (2011). Impact of the higher education reforms. Universities UK. 1 (2-4), 2-12.

Pahl, N., \& Richter, A. (2009). How To Gain Trust From Employees. GRIN Verlag.

Dan P. McCauley, Karl W. Kuhnert,(1992), A theoretical Review and Empirical investigation of Employee Trust in Management.

Tammy A. Boe (2002). Gaining and/or Maintaining Trust in Service Organization

STUC, 2009, Employee Trust and Motivation

Asherman, et. al(2000). "Building trust across cultural boundaries", Regulatory Affairs Focus, pp. 7-10, available at www.itapint1.com/PDF_boundaries.pdf; www.itapintl.com/inndex.php/aboutus/articles/building-trust-across-cultural-boundaries.

Askvik, S. (2010). The dynamics of political trust in South Africa, 1995-2006. Politikon, 37, $25-44$. doi:10.1080/ 02589346.2010.492148

House R.J, Hanges P.J, Ruiz- Quintanilla S.A and Dorfman P.W. (2000). Cultural influences on leadership and organizations: project GLOBE. Project GLOBE publications. 1 (1), 4-24. 